\title{
A Robust Position Control for Induction Machines
}

\author{
Oscar Barambones, Jose Maria Gonzalez de Durana and Luis Miguel Camarero
}

\begin{abstract}
A robust position control for induction motors using field oriented control theory is presented. The proposed controller is based on variable structure control and provides global asymptotic position tracking in the presence of unknown parameters and load torque variations. The proposed design incorporates an improved method of flux estimation that operates on the principle of flux and current observer. The proposed observer is basically an estimator that uses a plant model and a feedback loop with measured stator voltages and currents. The stability analysis of the proposed controller under parameter uncertainties and load disturbances is provided using the Lyapunov stability theory. Finally simulated results show that the proposed controller with the proposed observer provides high-performance dynamic characteristics and that this scheme is robust with respect to plant parameter variations and external load disturbances
\end{abstract}

\section{INTRODUCTION}

Induction motor position control is often used in some applications of electrical drives like robotic systems, conveyor belts, etc. In these applications uncertainty and external disturbances are present and therefore a robust control system that maintain the desired control performance under this situations are frequently required. Some control techniques have been developed to regulate these induction motors servo drives in high-performance applications. One of the most popular technique is the field oriented control method (Vas 1994, Leonhard 1996, Bose 2001).

The field-oriented technique guarantees the decoupling of torque and flux control commands of the induction motor, so that the induction motor can be controlled linearly as a separated excited D.C. motor. However, the control performance of the resulting linear system is still influenced by uncertainties, which usually are composed of unpredictable parameter variations, external load disturbances, and unmodelled and nonlinear dynamics.

In order to preserve the performance under these uncertainties, the variable structure control strategy using the slidingmode has been focussed on many studies and research for the the induction motor control (Cecati 2000, Benchaib 2000, Wang 2001, Barambones 2004, Wang 2005). The slidingmode control can offer many good properties, such as good performance against unmodelled dynamics, insensitivity to parameter variations, external disturbance rejection and fast dynamic response (Utkin 1993). These advantages of the

The authors are very grateful to the Basque Government by the support of this work through the project S-PE09UN12 and to the UPV/EHU by its support through projects GUI07/08.

Oscar Barambones and Jose Maria Gonzalez de Durana are with the Department of Automatic Control and System Engineering and Luis Miguel Camarero is with the Department of Chemical Engineering and Environment from the University of the Basque Country. EUI de Vitoria. Nieves cano 12, 01006 Vitoria, Spain. (phone: +34 945013235; email: oscar.barambones@ehu.es ). sliding-mode control may be employed in the position and speed control of an AC servo system.

This paper presents a variable estructure vector control scheme consisting, on the one hand of a flux estimation algorithm in order to avoid the flux sensors, and on the other hand, of a variable structure control algorithm that overcome the system uncertainties and load disturbances.

The flux estimation algorithm is based on a Luenberger observer and utilizes the measured stator voltages and currents values. The observer uses a plant model and incorporates a feedback loop with the estimation current error in order to overcome the system model uncertainties.

This manuscript is organized as follows. The flux observer is introduced in Section 2. Then, the proposed variable structure robust position control is presented in Section 3. In the Section 4 , some simulation results are presented. Finally, concluding remarks are stated in Section 5.

\section{ROTOR FLUX ESTIMATOR}

Many schemes (Lehonhard 1996, Bose 2001) based on simplified motor models have been devised to estimate some internal variables of the induction motor from measured terminal quantities. This procedure is frequently used in order to avoid the presence of some sensors in the control scheme. In order to obtain an accurate dynamic representation of the motor, it is necessary to base the calculation on the coupled circuit equations of the motor.

Since the motor voltages and currents are measured in a stationary frame of reference, it is also convenient to express the induction motor dynamical equations in this stationary frame.

The rotor voltage equations in the stationary frame may be written as (Bose 2001):

$$
\begin{aligned}
& v_{d r}=0=R_{r} i_{d r}+\dot{\psi}_{d r}+w_{r} \psi_{q r} \\
& v_{q r}=0=R_{r} i_{q r}+\dot{\psi}_{q r}-w_{r} \psi_{d r}
\end{aligned}
$$

where $\psi$ is the flux linkage; $v$ is the voltage; $R$ is the resistance, $i$ is the current and $w_{r}$ is the rotor electrical speed. The subscript $r$ denotes the rotor values refereed to the stator, and the subscripts $d$ and $q$ denote the dq-axis components in the stationary reference frame.

The stator voltage equations in the stationary frame may be written as:

$$
\begin{aligned}
& v_{d s}=R_{s} i_{d s}+\dot{\psi}_{d s} \\
& v_{q s}=R_{s} i_{q s}+\dot{\psi}_{q s}
\end{aligned}
$$

where the subscript $s$ denotes the stator values. 
On the other hand, the flux linkage expressions can be written in terms of currents and inductances:

$$
\begin{aligned}
& \psi_{d r}=L_{m} i_{d s}+L_{r} i_{d r} \\
& \psi_{q r}=L_{m} i_{q s}+L_{r} i_{q r} \\
& \psi_{d s}=L_{m} i_{d r}+L_{s} i_{d s} \\
& \psi_{q s}=L_{m} i_{q r}+L_{s} i_{q s}
\end{aligned}
$$

where $L_{m}$ is the magnetizing inductance, $L_{r}$ is the rotor inductance refereed to the stator and $L_{s}$ is the stator inductance.

Eliminating $i_{d r}$ and $i_{q r}$ from equations (1) and (2) respectively with the help of equations (5) and (6) yields:

$$
\begin{aligned}
& \dot{\psi}_{d r}=-\frac{R_{r}}{L_{r}} \psi_{d r}-w_{r} \psi_{q r}+\frac{L_{m} R_{r}}{L_{r}} i_{d s} \\
& \dot{\psi}_{q r}=-\frac{R_{r}}{L_{r}} \psi_{q r}+w_{r} \psi_{d r}+\frac{L_{m} R_{r}}{L_{r}} i_{q s}
\end{aligned}
$$

Eliminating $i_{d r}$ and $i_{q r}$ from equations (7) and (8) with the help of equations (5) and (6) respectively, we can find:

$$
\begin{aligned}
\psi_{d s} & =\frac{L_{m}}{L_{r}} \psi_{d r}+L_{s} \sigma i_{d s} \\
\psi_{q s} & =\frac{L_{m}}{L_{r}} \psi_{q r}+L_{s} \sigma i_{q s}
\end{aligned}
$$

were $\sigma=1-L_{m}^{2} /\left(L_{r} L_{s}\right)$ is the motor leakage coefficient.

now, substituting equations (11) and (12) in equations (3) and (4) and finding $\psi_{d r}$ and $\psi_{q r}$ it is obtained:

$$
\begin{aligned}
\dot{\psi}_{d r} & =\frac{L_{r}}{L_{m}} v_{d s}-\frac{L_{r}}{L_{m}} R_{s} i_{d s}-\frac{L_{r}}{L_{m}} \sigma L_{s} \dot{i}_{d s} \\
\dot{\psi}_{q r} & =\frac{L_{r}}{L_{m}} v_{q s}-\frac{L_{r}}{L_{m}} R_{s} i_{q s}-\frac{L_{r}}{L_{m}} \sigma L_{s} \dot{i}_{q s}
\end{aligned}
$$

Substituting equations (9) and (10) in equations (13) and (14), respectively, and finding $\dot{i}_{d s}$ and $\dot{i}_{q s}$, we get:

$$
\begin{gathered}
\dot{i}_{d s}=-\frac{L_{m}^{2} R_{r}+L_{r}^{2} R_{s}}{\sigma L_{s} L_{r}^{2}} i_{d s}+\frac{L_{m} R_{r}}{\sigma L_{s} L_{r}^{2}} \psi_{d r} \\
+\frac{L_{m} w_{r}}{\sigma L_{s} L_{r}} \psi_{q r}+\frac{1}{\sigma L_{s}} v_{d s} \\
\dot{i}_{q s}=-\frac{L_{m}^{2} R_{r}+L_{r}^{2} R_{s}}{\sigma L_{s} L_{r}^{2}} i_{q s}+\frac{L_{m} R_{r}}{\sigma L_{s} L_{r}^{2}} \psi_{q r} \\
-\frac{L_{m} w_{r}}{\sigma L_{s} L_{r}} \psi_{d r}+\frac{1}{\sigma L_{s}} v_{q s}
\end{gathered}
$$

Equations (15), (16), (9) and (10) constitute the desired state equations, which can be written in the form:

$$
\dot{x}=A x+B v_{s}
$$

where

$$
\begin{aligned}
x & =\left[\begin{array}{llll}
i_{d s} & i_{q s} & \psi_{d r} & \psi_{q r}
\end{array}\right]^{T} \\
v_{s} & =\left[\begin{array}{llll}
v_{d s} & v_{q s} & 0 & 0
\end{array}\right]^{T} \\
B & =\left[\begin{array}{cccc}
\frac{1}{\sigma L_{s}} & 0 & 0 & 0 \\
0 & \frac{1}{\sigma L_{s}} & 0 & 0
\end{array}\right]^{T} \\
A & =\left[\begin{array}{cccc}
-\rho & 0 & \frac{R_{r}}{c L_{r}} & \frac{w_{r}}{c} \\
0 & -\rho & -\frac{w_{r}}{c} & \frac{R_{r}}{c L_{r}} \\
\frac{L_{m} R_{r}}{L_{r}} & 0 & -\frac{R_{r}}{L_{r}} & -w_{r} \\
0 & \frac{L_{m} R_{r}}{L_{r}} & w_{r} & -\frac{R_{r}}{L_{r}}
\end{array}\right] \\
\text { where } \rho & =\frac{L_{m}^{2} R_{r}+L_{r}^{2} R_{s}}{\sigma L_{s} L_{r}^{2}} \text { and } c=\frac{\sigma L_{s} L_{r}}{L_{m}} .
\end{aligned}
$$

Considering the stator currents as the system output, the output equation for this system is:

$$
y=C x
$$

where

$$
C=\left[\begin{array}{llll}
1 & 0 & 0 & 0 \\
0 & 1 & 0 & 0
\end{array}\right]
$$

The states observer, which estimates the system states (stator current and rotor flux), is defined by means of the following equation (Luenberger observer):

$$
\begin{aligned}
\dot{\hat{x}} & =A \hat{x}+B v_{s}+G(y-C \hat{x}) \\
& =A \hat{x}+B v_{s}+G C(x-\hat{x})
\end{aligned}
$$

where the symbol $(\hat{\cdot})$ represents the estimated values and $G$ is the observer gain matrix.

The estimation error of the system states (stator currents and rotor fluxes) may be obtained subtracting equations (17) and (20) as:

$$
\dot{x}-\dot{\hat{x}}=A x-A \hat{x}-G C(x-\hat{x})
$$

Then, the following estimation error dynamic is obtained:

$$
\dot{\tilde{x}}=(A-G C) \tilde{x}
$$

where $\tilde{x}=x-\hat{x}$ is the state estimation error.

Therefore, if the observer gain $G$ is chosen such that the characteristic equation of the matrix $A-G C$ has all its roots with a negative real part, then the estimation error converges to zero. Consequently the estimated states $\hat{i}_{d s}, \hat{i}_{q s}, \hat{\psi}_{d s}, \hat{\psi}_{q s}$ converges to the real states $i_{d s}, i_{q s}, \psi_{d s}, \psi_{q s}$ as $t$ tends to infinity. Hence, the rotor flux may be obtained from the state observer given by equation (19). 


\section{VARIABLE STRUCTURE ROBUST POSITION CONTROL}

The mechanical equation of an induction motor can be written as:

$$
J \ddot{\theta}_{m}+B \dot{\theta}_{m}+T_{L}=T_{e}
$$

where $J$ and $B$ are the inertia constant and the viscous friction coefficient of the induction motor respectively; $T_{L}$ is the external load; $\theta_{m}$ is the rotor mechanical position, which is related to the rotor electrical position, $\theta_{r}$, by $\theta_{m}=2 \theta_{r} / p$ where $p$ is the pole numbers and $T_{e}$ denotes the generated torque of an induction motor, defined as (Bose 2001):

$$
T_{e}=\frac{3 p}{4} \frac{L_{m}}{L_{r}}\left(\psi_{d r}^{e} i_{q s}^{e}-\psi_{q r}^{e} i_{d s}^{e}\right)
$$

where $\psi_{d r}^{e}$ and $\psi_{q r}^{e}$ are the rotor-flux linkages, with the subscript ' $\mathrm{e}$ ' denoting that the quantity is refereed to the synchronously rotating reference frame; $i_{q s}^{e}$ and $i_{d s}^{e}$ are the stator currents, and $p$ is the pole numbers.

The relation between the synchronously rotating reference frame and the stationary reference frame is performed by the so-called reverse Park's transformation:

$$
\left[\begin{array}{l}
x_{a} \\
x_{b} \\
x_{c}
\end{array}\right]=\left[\begin{array}{cc}
\cos \left(\theta_{e}\right) & -\sin \left(\theta_{e}\right) \\
\cos \left(\theta_{e}-2 \pi / 3\right) & -\sin \left(\theta_{e}-2 \pi / 3\right) \\
\cos \left(\theta_{e}+2 \pi / 3\right) & -\sin \left(\theta_{e}+2 \pi / 3\right)
\end{array}\right]\left[\begin{array}{l}
x_{d} \\
x_{q}
\end{array}\right]
$$

where $\theta_{e}$ is the angle position between the d-axis of the synchronously rotating reference frame and the a-axis of the stationary reference frame, and it is assumed that the quantities are balanced.

The estimated angular position of the rotor flux vector $\left(\bar{\psi}_{r}\right)$ related to the d-axis of the stationary reference frame may be calculated by means of the rotor flux components in this reference frame $\left(\hat{\psi}_{d r}, \hat{\psi}_{q r}\right)$ as follows:

$$
\hat{\theta}_{e}=\arctan \left(\frac{\hat{\psi}_{q r}}{\hat{\psi}_{d r}}\right)
$$

where $\hat{\theta}_{e}$ is the estimated angular position of the rotor flux vector.

Using the field-orientation control principle (Bose 2001), the current component $i_{d s}^{e}$ is aligned in the direction of the rotor flux vector $\bar{\psi}_{r}$, and the current component $i_{q s}^{e}$ is aligned in the perpendicular direction to it. At this condition, it is satisfied that:

$$
\psi_{q r}^{e}=0, \quad \psi_{d r}^{e}=\left|\bar{\psi}_{r}\right|
$$

Taking into account the results presented in equation (27), the equation of induction motor torque (24) is simplified to:

$$
T_{e}=\frac{3 p}{4} \frac{L_{m}}{L_{r}} \psi_{d r}^{e} i_{q s}^{e}=K_{T} i_{q s}^{e}
$$

where $K_{T}$ is the torque constant, defined as follows:

$$
K_{T}=\frac{3 p}{4} \frac{L_{m}}{L_{r}} \psi_{d r}^{e^{*}}
$$

where $\psi_{d r}^{e^{*}}$ denotes the command rotor flux.

With the above mentioned proper field orientation, the dynamics of the rotor flux is given by (Bose 2001):

$$
\frac{d \psi_{d r}^{e}}{d t}+\frac{\psi_{d r}^{e}}{T_{r}}=\frac{L_{m}}{T_{r}} i_{d s}^{e}
$$

Then, the mechanical equation (23) becomes:

$$
\ddot{\theta}_{m}+a \dot{\theta}_{m}+f=b i_{q s}^{e}
$$

where the parameter are defined as:

$$
a=\frac{B}{J}, \quad b=\frac{K_{T}}{J}, \quad f=\frac{T_{L}}{J} ;
$$

Now, we are going to consider the previous mechanical equation (31) with uncertainties as follows:

$$
\ddot{\theta}_{m}=-(a+\triangle a) \dot{\theta}_{m}-(f+\triangle f)+(b+\triangle b) i_{q s}^{e}
$$

where the terms $\triangle a, \triangle b$ and $\triangle f$ represents the uncertainties of the terms $a, b$ and $f$ respectively.

Let us define the position tracking error as follows:

$$
e(t)=\theta_{m}(t)-\theta_{m}^{*}(t)
$$

where $\theta_{m}^{*}$ is the rotor position command.

Taking the second derivative of the previous equation with respect to time yields:

$$
\ddot{e}(t)=\ddot{\theta}_{m}-\ddot{\theta}_{m}^{*}=-a e(t)+u(t)+d(t)
$$

where the following terms have been collected in the signal $u(t)$,

$$
u(t)=b i_{q s}^{e}(t)-a \dot{\theta}_{m}^{*}(t)-f(t)-\ddot{\theta}_{m}^{*}(t)
$$

and the uncertainty terms have been collected in the signal $d(t)$,

$$
d(t)=-\triangle a w_{m}(t)-\triangle f(t)+\triangle b i_{q s}^{e}(t)
$$

Now, we are going to define the sliding variable $S(t)$ as:

$$
S(t)=\dot{e}(t)+k e(t)
$$

where $k$ is a positive constant gain.

Then, the sliding surface is defined as:

$$
S(t)=\dot{e}(t)+k e(t)=0
$$

The variable structure speed controller is designed as:

$$
u(t)=-(k-a) \dot{e}(t)-\beta \operatorname{sgn}(S)
$$

where the $k$ is the previously defined gain, $\beta$ is the switching gain, $S$ is the sliding variable defined in eqn. (38) and $\operatorname{sgn}(\cdot)$ is the sign function.

In order to obtain the speed trajectory tracking, the following assumption should be formulated:

( $\mathcal{A} 1)$ The gain $\beta$ must be chosen so that $\beta \geq \bar{d}$ where $\bar{d} \geq \sup _{t \in R^{0+}}|d(t)|$. 
Note that this condition only implies that the system uncertainties are bounded magnitudes.

Theorem 1: Consider the induction motor given by equation (33). Then, if the assumption $(\mathcal{A} 1)$ are verified, the control law (40) leads the rotor mechanical position $\theta_{m}(t)$ so that the position tracking error $e(t)=\theta_{m}(t)-\theta_{m}^{*}(t)$ tends to zero as the time tends to infinity.

The proof of this theorem will be carried out using the Lyapunov stability theory.

Proof: Define the Lyapunov function candidate:

$$
V(t)=\frac{1}{2} S(t) S(t)
$$

Its time derivative is calculated as:

$$
\begin{aligned}
\dot{V}(t) & =S(t) \dot{S}(t) \\
& =S \cdot[\ddot{e}+k \dot{e}] \\
& =S \cdot[(-a \dot{e}+u+d)+k \dot{e}] \\
& =S \cdot[(k-a) \dot{e}+u+d] \\
& =S \cdot[(k-a) \dot{e}-(k-a) \dot{e}-\beta \operatorname{sgn}(S)+d] \\
& =S \cdot[d-\beta \operatorname{sgn}(S)] \\
& \leq-(\beta-|d|)|S| \\
& \leq 0
\end{aligned}
$$

It should be noted that the eqns. (38), (35) and (40), and the assumption $(\mathcal{A} 1)$ have been used in the proof.

Using the Lyapunov's direct method, since $V(t)$ is clearly positive-definite, $\dot{V}(t)$ is negative definite and $V(t)$ tends to infinity as $S(t)$ tends to infinity, then the equilibrium at the origin $S(t)=0$ is globally asymptotically stable. Therefore $S(t)$ tends to zero as the time $t$ tends to infinity. Moreover, all trajectories starting off the sliding surface $S=0$ must reach it in finite time and then they will remain on this surface. This system's behavior, once on the sliding surface is usually called sliding mode (Utkin 1993).

When the sliding mode occurs on the sliding surface (39), then $S(t)=0$, and therefore the dynamic behavior of the tracking problem (35) is equivalently governed by the following equation:

$$
\dot{S}(t)=0 \quad \Rightarrow \quad \dot{e}(t)=-k e(t)
$$

Then, like $k$ is a positive constant, the tracking error $e(t)$ and its derivative $\dot{e}(t)$ converges to zero exponentially.

It should be noted that, a typical motion under sliding mode control consists of a reaching phase during which trajectories starting off the sliding surface $S=0$ move toward it and reach it in finite time, followed by sliding phase during which the motion will be confined to this surface and the system tracking error will be represented by the reduced-order model (43), where the tracking error tends to zero.
Finally, the torque current command, $i_{q s}^{*}(t)$, can be obtained directly substituting eqn. (40) in eqn. (36):

$$
i_{q s}^{*}(t)=\frac{1}{b}\left[-(k-a) \dot{e}-\beta \operatorname{sgn}(S)+a \theta_{m}^{*}+\dot{\theta}_{m}^{*}+f(t)\right]
$$

It should be noted that the current command is a bounded signal because all its components are bounded.

Therefore, the proposed variable structure position control resolves the speed tracking problem for the induction motor in presence of some uncertainties in mechanical parameters and load torque.

\section{Simulation Results}

In this section we will study the position regulation performance of the proposed sliding-mode field oriented control versus reference and load torque variations by means of simulation examples.

The block diagram of the proposed robust position control scheme is presented in Figure 1. The function of the blocks that appear in this figure are:

The block 'VSC Controller' represents the proposed slidingmode controller, and it is implemented by equations (38) and (44). The block 'limiter' limits the current applied to the motor windings so that it remains within the limit value, being implemented by a saturation function. The block ' $d q^{e} \rightarrow a b c$ ' makes the conversion between the synchronously rotating and stationary reference frames, and it is implemented by equation (25). The block 'Current Controller' consists of a three hysteresis-band current PWM control, which is basically an instantaneous feedback current control method of PWM where the actual current $\left(i_{a b c}\right)$ continually tracks the command current $\left(i_{a b c}^{*}\right)$ within a hysteresis band. The block 'PWM Inverter' is a six IGBT-diode bridge inverter with $780 \mathrm{~V} \mathrm{DC}$ voltage source. The block 'Field Weakening' gives the flux command based on rotor speed, so that the PWM controller does not saturate. The block ' $i_{d s}^{e *}$ Calculation' provides the current reference $i_{d s}^{e *}$ from the rotor flux reference through the equation (30). The block 'Flux Estimator' represents the proposed Fux estimator, and it is implemented by the equation (19). The block ' $\hat{\theta}_{e}$ Calculation' provides the angular position of the rotor flux vector. Finally, the block 'IM' represents the induction motor.

The induction motor used in this case study is a $50 \mathrm{HP}, 460$ $\mathrm{V}$, four pole, $60 \mathrm{~Hz}$ motor having the following parameters: $R_{s}=0.087 \Omega, R_{r}=0.228 \Omega, L_{s}=35.5 \mathrm{mH}, L_{r}=$ $35.5 \mathrm{mH}$, and $L_{m}=34.7 \mathrm{mH}$.

The system has the following mechanical parameters: $J=$ $1.662 \mathrm{~kg} . \mathrm{m}^{2}$ and $B=0.1 \mathrm{~N} . \mathrm{m} . \mathrm{s}$. It is assumed that there is an uncertainty around $20 \%$ in the system parameters, that will be overcome by the proposed sliding control.

The following values have been chosen for the state ob- 


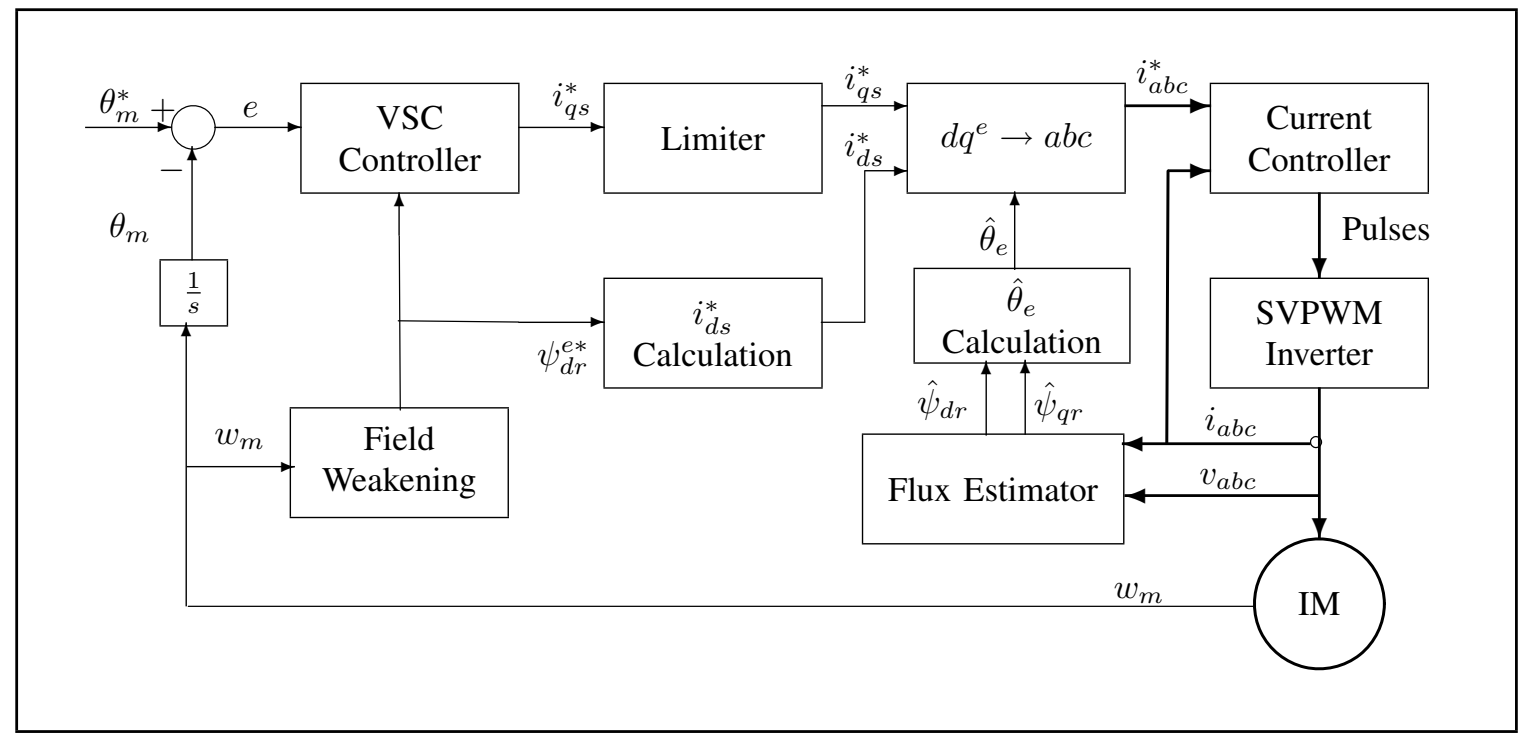

Fig. 1. Block diagram of the proposed sliding-mode field oriented control

server:

$$
G=\left[\begin{array}{cc}
100 & 0 \\
0 & 100 \\
0 & -1 \\
1 & 0
\end{array}\right]
$$

Finally, the following values have been chosen for the controller parameters, $k=100, \beta=35$.

In this example the motor starts from a standstill state and we want the rotor position to follow a position command that starts from zero and finish at $\theta_{m}^{*}=2.5 \mathrm{rad}$ following a soft reference. The system starts with an initial load torque $T_{L}=$ 100 N.m, and at time $t=1.5 \mathrm{~s}$, the load torque steps from $T_{L}=100 \mathrm{~N} . \mathrm{m}$ to $T_{L}=250 \mathrm{~N} . \mathrm{m}$.

Figure 2 shows the desired rotor position (dashed line) and the real rotor position (solid line). As it may be observed, the rotor position track the desired position in spite of system uncertainties. Moreover, the position tracking is not affected by the load torque change at time $t=1.5 \mathrm{~s}$, because when the sliding surface is reached (sliding mode) the system becomes insensitive to the boundary external disturbances.

Figure 3 shows rotor speed. As it may be observed, the rotor speed increases until time $t=0.17 \mathrm{~s}$ and then decreases until time $t=0.8 \mathrm{~s}$ when the speed reach the zero value. Then, the speed is maintained at zero value in spite of the load torque change at time $t=1.5$.

Figure 4 shows the current of one stator winding. It may be observed that in the initial state, the current signal presents a high value because it is necessary a high torque to increment the rotor speed. In the constant speed region, the motor torque only has to compensate the friction and the load torque and so, the current amplitude is smaller. Finally, at time $t=1.5 \mathrm{~s}$ the current increases because the load torque has been increased.

Figure 5 shows the motor torque. As in the case of the current (fig. 4), the motor torque has a high initial value in the speed acceleration zone, then the value decreases in the deceleration zone, and finally increases due to the load torque increment. In this figure it may be seen that in the motor torque appears the so-called chattering phenomenon, however this high frequency changes in the torque will be filtered by the mechanical system inertia. Nevertheless, the chattering can be reduced changing the sign function present in the control law (44) by a saturation function (Barambones 2002).

\section{CONCLUSION}

In this paper a induction motor position regulation ussing a sliding mode vector control has been presented. It is also 


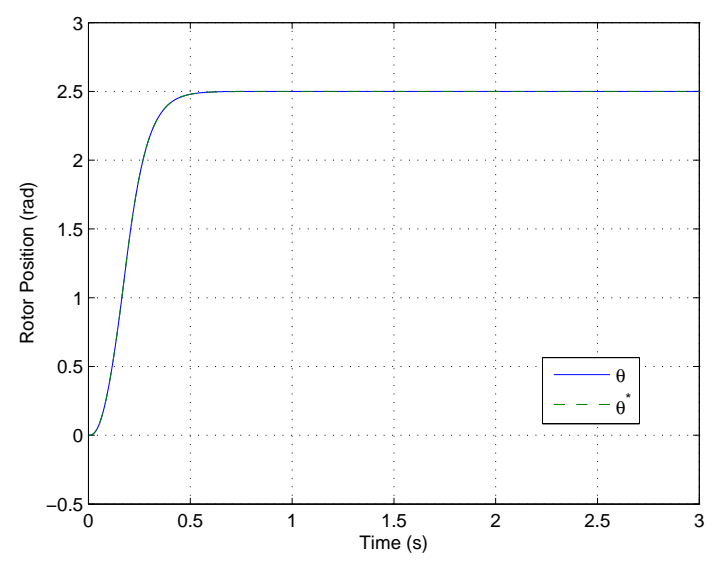

Fig. 2. Reference and real rotor position signals (rad)

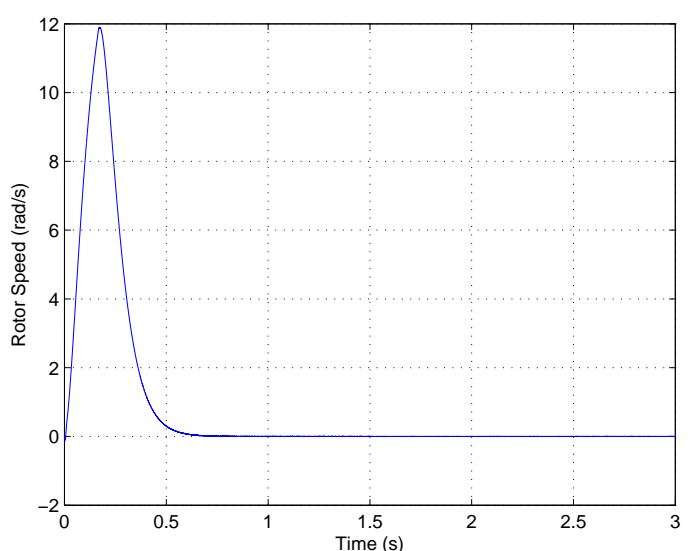

Fig. 3. Reference and real rotor speed signals ( $\mathrm{rad} / \mathrm{s})$

proposed a flux estimator that overcomes the flux sensors. The flux estimation algorithm is based on a Luenberger observer and employs the measured stator voltages and currents in the stationary reference frame.

Due to the nature of the sliding mode control this control scheme is robust under uncertainties caused by parameter error or by changes in the load torque. The closed loop stability of the presented design has been proved through Lyapunov stability theory.

Finally, by means of simulation examples, it has been shown that the proposed position control scheme performs reasonably well in practice, and that the speed tracking objective is achieved under uncertainties in the parameters and under load torque variations.

\section{REFERENCES}

[1] BARAmbones O. AND ETXEBARRIA V., 2002, Robust neural control for robotic manipulators, Automatica, 38, pp.235-242.

[2] Barambones, O. And Garrido, A.J., 2004, A sensorless variable structure control of induction motor drives, Electric Power Systems Research, 72, 21-32.

[3] Bose, B.K., 2001, Modern Power Electronics and AC Drives., Prentice Hall, New Jersey.

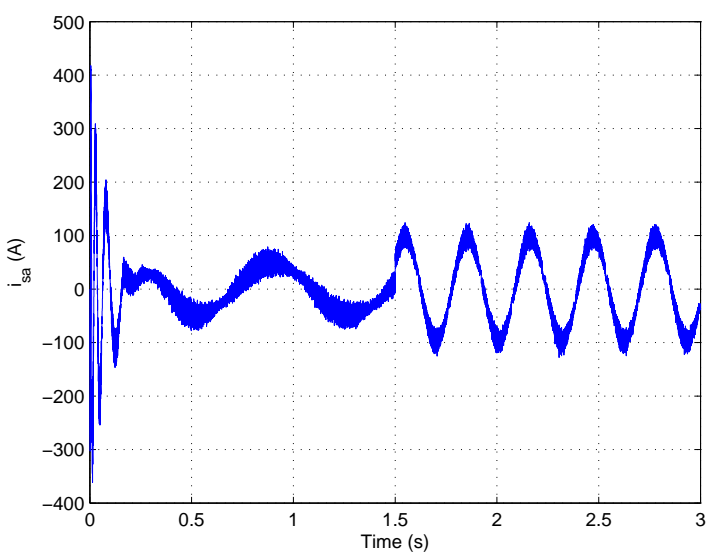

Fig. 4. Stator Current $i_{s a}$ (A)

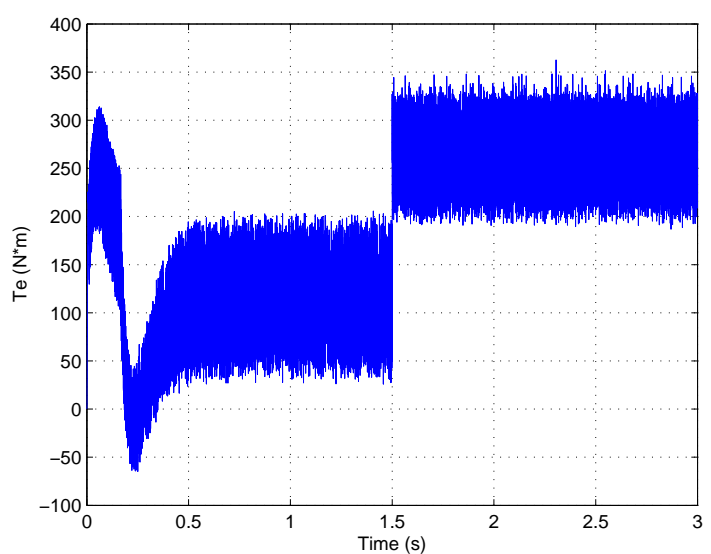

Fig. 5. Motor torque (N.m)

[4] Cecati,2000, Position control of the induction motor using a passivitybased controller. IEEE Trans. on Industry Applications, 36, 1277-1284.

[5] Chern, T.L., ChAng, J. AND TSAI, K.L.,1998, Integral variable structure control based adaptive speed estimator and resistance identifier for an induction motor. Int. J. of Control, 69, 31-47.

[6] Lehonhard, W., 1996, Control of Electrical Drives. Springer, Berlin.

[7] LIN, F.K. AND LIAW, C.M.,1993, Control of indirect field-oriented induction motor drives considering the effects of dead-time and parameter variations. IEEE Trans. Indus. Electro, 40, 486-495.

[8] UTKIN V.I., 1993, Sliding mode control design principles and applications to electric drives, IEEE Trans. Indus. Electro., 40, 26-36.

[9] VAS, P., 1994, Vector Control of AC Machines. Oxford Science Publications, Oxford.

[10] WANG, W.J. AND CHEN J.Y.,2001, Compositive adaptive position control of induction motors based on pasivity theory IEEE Trans. on Energy conversion, 16, 180-185.

[11] WANG, W.J. AND CHEN J.Y.,2005, Passivity-based sliding mode position control for induction motor drives IEEE Trans. on Energy conversion, 20, 316-321. 